\title{
Should ondansetron use be a reason to admit children with isolated, nondisplaced, linear skull fractures?
}

\author{
Jonathan Dallas, BS, ${ }^{1,3}$ Evan Mercer, BA, ${ }^{1}$ Rebecca A. Reynolds, MD, ${ }^{2,3}$ \\ John C. Wellons III, MD, MSPH, ${ }^{2,3}$ Chevis N. Shannon, DrPH, MBA, ${ }^{2,3}$ and \\ Christopher M. Bonfield, MD2,3
}

${ }^{1}$ Vanderbilt University School of Medicine; 'Department of Neurosurgery, Vanderbilt University Medical Center; and ${ }^{3}$ Surgical
Outcomes Center for Kids, Monroe Carell Jr. Children's Hospital at Vanderbilt, Nashville, Tennessee

OBJECTIVE Isolated, nondisplaced skull fractures (ISFs) are a common result of pediatric head trauma. They rarely require surgical intervention; however, many patients with these injuries are still admitted to the hospital for observation. This retrospective study investigates predictors of vomiting and ondansetron use following pediatric ISFs and the role that these factors play in the need for admission and emergency department (ED) revisits.

METHODS The authors identified pediatric patients (< 18 years old) with a linear ISF who had presented to the ED of a single tertiary care center between 2008 and 2018. Patients with intracranial hemorrhage, significant fracture displacement, or other traumatic injuries were excluded. Outcomes included vomiting, ondansetron use, admission, and revisit following ED discharge. Both univariable and multivariable analyses were used to determine significant predictors of each outcome $(p<0.05)$.

RESULTS Overall, 518 patients were included in this study. The median patient age was 9.98 months, and a majority of the patients (59\%) were male. The most common fracture locations were parietal $(n=293[57 \%])$ and occipital $(n=144$ [28\%]). Among the entire patient cohort, 124 patients (24\%) had documented vomiting, and 64 of these patients $(52 \%)$ received ondansetron. In a multivariable analysis, one of the most significant predictors of vomiting was occipital fracture location (OR 4.05, $p<0.001)$. In turn, and as expected, both vomiting (OR 14.42, $p<0.001$ ) and occipital fracture location (OR 2.66, $p=0.017$ ) were associated with increased rates of ondansetron use. A total of 229 patients $(44 \%)$ were admitted to the hospital, with vomiting as the most common indication for admission ( $n=59$ [26\%]). Moreover, $4.1 \%$ of the patients had ED revisits following initial discharge, and the most common reason was vomiting (11/21 [52\%]). Howev$\mathrm{er}$, in the multivariable analysis, ondansetron use at initial presentation (and not vomiting) was the sole predictor of revisit following initial ED discharge (OR 5.05, $p=0.009$ ).

CONCLUSIONS In this study, older patients and those with occipital fractures were more likely to present with vomiting and to be treated with ondansetron. Additionally, ondansetron use at initial presentation was found to be a significant predictor of revisits following ED discharge. Ondansetron could be masking recurrent vomiting in ED patients, and this should be considered when deciding which patients to observe further or discharge.

https://thejns.org/doi/abs/10.3171/2019.9.PEDS19203

KEYWORDS skull fracture; vomiting; ondansetron; hospital admission; revisit; trauma

$\mathrm{S}$ KULL fractures are a common reason for presentation to the pediatric emergency department (ED), but there is significant variability in injury severity. A number of studies have shown that isolated, nondisplaced skull fractures (ISFs) pose little to no risk of neurological decline and rarely require further intervention other than brief ED observation. ${ }^{3-6,9,11,14-16,18-20}$ Despite this low risk, however, many patients are still admitted to the hospital for reasons such as young age or positive imaging findings and are discharged shortly thereafter (generally within 1 or 2 days). 1,3,4,6,11,12,14,16,19,20 Such unnecessary admissions have been shown to result in significantly higher treatment costs with little medical benefit. ${ }^{11,16,20}$

Potential solutions to this problem have been explored

ABBREVIATIONS CARE = Child Protection and Well-Being; ED = emergency department; ISF = isolated, nondisplaced skull fracture; NAT = nonaccidental trauma; $P O=$ per os.

SUBMITTED April 17, 2019. ACCEPTED September 30, 2019.

INCLUDE WHEN CITING Published online December 13, 2019; DOI: 10.3171/2019.9.PEDS19203. 
in prior studies. Some hospitals have incorporated protocols to determine which patients actually require admission to the hospital, using metrics such as the Glasgow Coma Scale or other presenting symptoms to determine discharge safety. ${ }^{12,16}$ Other researchers have argued that hospitals should only admit patients with ISFs in the presence of neurological deficits, recurrent symptoms, or suspected nonaccidental trauma (NAT)., ${ }^{1,3,15,16,19}$ Ultimately, the aims of these studies were to decrease the rate of unnecessary skull fracture admissions and to reduce costs for both the patient and the hospital.

Despite these efforts, there is still no well-established protocol to determine which patients require admission and which can be safely discharged home. Given anecdotal experience, we suspected that a higher incidence of nausea and vomiting is associated with occipital skull fractures; however, this hypothesis has never been scientifically tested. We also hypothesized that a history of nausea and vomiting is a predictor of initial hospital admission and subsequent ED revisit. It was further suspected that there may be a discordant use of ondansetron for prophylaxis and a documented history of vomiting; thus, these two variables were classified separately for each patient in the current study. The aims of this study were threefold. First, we determined if occipital fracture location and other patient-related factors (such as sociodemographics and injury profile) are associated with nausea, vomiting, and ondansetron use following ISF. Second, we determined predictors of admission for such patients at our institution. Last, we investigated risk factors for ED revisits after discharge.

\section{Methods \\ Patient Cohort}

In this retrospective cohort study, we searched a deidentified patient database for pediatric patients with an ISF who had presented to the ED of Monroe Carell Jr. Children's Hospital at Vanderbilt in the period from July 2008 to June 2018. Inclusion criteria selected for all patients with an ICD-9 or ICD-10 code for skull fracture. Patients were subsequently excluded if they 1 ) were at least 18 years of age, 2) had fracture displacement, 3) had intracranial hemorrhage, or 4) had other significant injuries as determined by both ICD codes and manual review. Patients with any intracranial blood, even a clinically silent amount, were excluded in order to capture only those patients who had been treated exclusively for skull fractures and associated concerns (e.g., NAT workup or social situations). Patients were also excluded if they had been admitted for an unrelated reason when they were injured (i.e., an inpatient fall).

\section{Study Variables and Outcomes}

The primary study outcomes were chosen to address each of the three study aims: patient-related predictors of nausea/vomiting and ondansetron use, hospital admission, and ED revisit. Nausea/vomiting and ondansetron use were classified separately because not all patients with documented vomiting received ondansetron and vice versa. Data collected for each patient included demograph- ics, injury/fracture information, symptoms, consults, and admission and discharge details. Demographics included age, sex, race, and ethnicity. Injury/fracture information included mechanism of injury, time from injury to presentation to our institution, fracture location, pneumocephalus, and CSF leakage. Symptoms included loss of consciousness, nausea/vomiting, and whether or not the patient received antiemetic medication (ondansetron). Logged consults included neurosurgery, trauma surgery, and the Child Protection and Well-Being (CARE) team. Finally, admission and discharge information (if applicable) included reason for admission, time until discharge, whether the patient re-presented to the ED within 72 hours, and if so, the reason for revisit.

\section{Statistical Analysis}

Data were stored in a secure Research Electronic Data Capture (REDCap) database. ${ }^{10}$ All statistical analysis was performed with a combination of Microsoft Excel (Microsoft Corp.) and SPSS (IBM Corp.) software. Analysis was performed to identify significant predictors of each primary outcome as detailed above. For each outcome, initial univariable analysis was performed using either Pearson's chi-square analysis or Fisher's exact test for categorical predictors or univariable logistic regression for continuous predictors. However, there is likely a significant amount of confounding between certain variables, such as nausea and ondansetron use, or mechanism of injury and hospital admission. Thus, all variables significant at a relaxed $p$ value in the univariable analysis $(\mathrm{p}<0.10)$ were included in a subsequent multivariable logistic regression. This was used to control for unmeasured confounding in the univariable analysis and identify truly significant predictors of each outcome. Statistical significance was set a priori at $\mathrm{p}<0.05$.

\section{Results}

Demographic information is summarized in Table 1. Overall, 518 patients presented to our ED with an ISF during the 10-year study period and met all study inclusion criteria, composing the study cohort. The median patient age was 9.98 months (IQR 5.81-30.44). Males outnumbered females at a ratio of approximately $3: 2$, and a majority of patients were white (76.1\%).

The most common mechanism of injury was a fall from $\leq 3$ feet $(62.4 \%)$, followed by falls from greater heights (13.7\%). Twelve percent of patients presented with no known mechanism of injury. A majority of patients (59.1\%) presented within 6 hours of injury, though many patients took longer. The most common fracture sites were parietal $(56.6 \%)$ and occipital $(27.8 \%)$. There were 30 cases of pneumocephalus (5.8\%), but none of these cases had any definitive CSF leakage. One hundred twenty-four patients (23.9\%) had documented vomiting; of those, 64 (51.6\%) received ondansetron at some point. Among the entire patient cohort, only 89 patients $(17.2 \%)$ received ondansetron.

Consult rates were as follows: $71.0 \%$ neurosurgery, $33.2 \%$ trauma surgery, and $15.1 \%$ CARE team. Forty-four percent of patients were admitted to the hospital, and most 


\section{TABLE 1. Descriptive statistics of the patient cohort}

\begin{tabular}{|c|c|}
\hline Variable & Value \\
\hline \multicolumn{2}{|l|}{ Age in mos } \\
\hline Mean (SD) & $24.36(31.50)$ \\
\hline Median (IQR) & $9.98(5.81-30.44)$ \\
\hline \multicolumn{2}{|l|}{ Sex, no. $(\%)$} \\
\hline Male & $307(59.3)$ \\
\hline Female & $211(40.7)$ \\
\hline \multicolumn{2}{|l|}{ Race, no. (\%) } \\
\hline African American & $59(11.4)$ \\
\hline Asian/Pacific Islander & $8(1.5)$ \\
\hline White & $394(76.1)$ \\
\hline Native American & $1(0.2)$ \\
\hline Multirace/other/unknown & $56(10.8)$ \\
\hline \multicolumn{2}{|l|}{ Ethnicity, no. (\%) } \\
\hline Hispanic/Latino & $50(9.7)$ \\
\hline Not Hispanic/Latino & $409(79.0)$ \\
\hline Unknown & $59(11.4)$ \\
\hline \multicolumn{2}{|l|}{ Mechanism of injury, no. (\%) } \\
\hline Fall $\leq 3 \mathrm{ft}$ & $323(62.4)$ \\
\hline Fall $>3 \mathrm{ft}$ & $71(13.7)$ \\
\hline MVC & $20(3.9)$ \\
\hline Unknown (presented w/ scalp swelling) & $62(12.0)$ \\
\hline Other (bike, ATV, blunt trauma) & $42(8.1)$ \\
\hline \multicolumn{2}{|l|}{ Time from injury to presentation, no. (\%) } \\
\hline$<6 \mathrm{hrs}$ & $306(59.1)$ \\
\hline $6-24 \mathrm{hrs}$ & $109(21.0)$ \\
\hline$>24 \mathrm{hrs}$ & $103(19.9)$ \\
\hline \multicolumn{2}{|l|}{ Principal location of fracture, no. (\%) } \\
\hline Occipital & $144(27.8)$ \\
\hline Temporal & $45(8.7)$ \\
\hline Frontal & $36(6.9)$ \\
\hline Parietal & $293(56.6)$ \\
\hline \multicolumn{2}{|l|}{ Loss of consciousness, no. (\%) } \\
\hline Yes & $29(5.6)$ \\
\hline No & $403(77.8)$ \\
\hline Unknown & $86(16.6)$ \\
\hline \multicolumn{2}{|l|}{ Pneumocephalus, no. (\%) } \\
\hline Yes & $30(5.8)$ \\
\hline No & $488(94.2)$ \\
\hline \multicolumn{2}{|l|}{$\begin{array}{l}\text { CSF leakage (pneumocephalus patients only), } \\
\text { no. (\%) }\end{array}$} \\
\hline Yes & $0(0.0)$ \\
\hline No & $30(100.0)$ \\
\hline \multicolumn{2}{|l|}{ Nausea/vomiting, no. (\%) } \\
\hline Yes & $124(23.9)$ \\
\hline No & $394(76.1)$ \\
\hline \multicolumn{2}{|l|}{ Ondansetron use, no. (\%) } \\
\hline Yes & $89(17.2)$ \\
\hline No & $429(82.8)$ \\
\hline
\end{tabular}

CONTINUED IN NEXT COLUMN »
» CONTINUED FROM PREVIOUS COLUMN

TABLE 1. Descriptive statistics of the patient cohort

\begin{tabular}{|c|c|}
\hline Variable & Value \\
\hline \multicolumn{2}{|l|}{ Neurosurgery consult, no. (\%) } \\
\hline Yes & $368(71.0)$ \\
\hline No & $150(29.0)$ \\
\hline \multicolumn{2}{|l|}{ Trauma consult, no. (\%) } \\
\hline Yes & $172(33.2)$ \\
\hline No & $346(66.8)$ \\
\hline \multicolumn{2}{|l|}{ CARE consult, no. (\%) } \\
\hline Yes & $78(15.1)$ \\
\hline No & $440(84.9)$ \\
\hline \multicolumn{2}{|c|}{ Hospital admission status, no. (\%) } \\
\hline Yes & $229(44.2)$ \\
\hline No & $289(55.8)$ \\
\hline \multicolumn{2}{|l|}{ Time until discharge, no. (\%) } \\
\hline Later the same day & $28(12.2)$ \\
\hline The following day & $180(78.6)$ \\
\hline 2 or more days later & $21(9.2)$ \\
\hline \multicolumn{2}{|c|}{ Reason for hospital admission, no. (\%) } \\
\hline Vomiting/PO challenge & $59(25.8)$ \\
\hline CARE team consultation & $54(23.6)$ \\
\hline Young age & $48(21.0)$ \\
\hline Other & $38(16.6)$ \\
\hline Unclear & $30(13.1)$ \\
\hline \multicolumn{2}{|l|}{ 72-hr ED revisit, no. (\%) } \\
\hline Yes & $21(4.1)$ \\
\hline No & $497(95.9)$ \\
\hline
\end{tabular}

ATV = all-terrain vehicle; $\mathrm{MVC}=$ motor vehicle collision.

of them (90.8\%) were discharged by the next day. Of the 21 patients who were kept in the hospital for more than 1 day, 8 had NAT suspicion and another 4 were admitted for monitoring of either pneumocephalus or other neurological symptoms. Reasons for admission were variable but most commonly included per os (PO) challenge (25.8\%), CARE consult (23.6\%), and young age (21.0\%; Fig. 1). Twenty-one patients (4.1\%) revisited the ED following their initial discharge (Fig. 2).

The statistical significance of each independent variable in the univariable analysis is shown in Table 2. All variables with a significance of $p<0.10$ were included in the multivariable analysis, the results of which are shown in Table 3. Following multivariable analysis, the only predictors of vomiting were increased age and occipital fracture location. Similarly, significant predictors of ondansetron use were increased age, motor vehicle collision, occipital or temporal fracture locations, and vomiting.

While PO challenge was the most common indication for admission to the hospital, neither vomiting (OR 1.88, $p=0.103)$ nor ondansetron use (OR 2.05, $p=0.123)$ was independently predictive of admission. Consult to any of the services listed above (neurosurgery, trauma surgery, CARE) was associated with a significantly increased ad- 


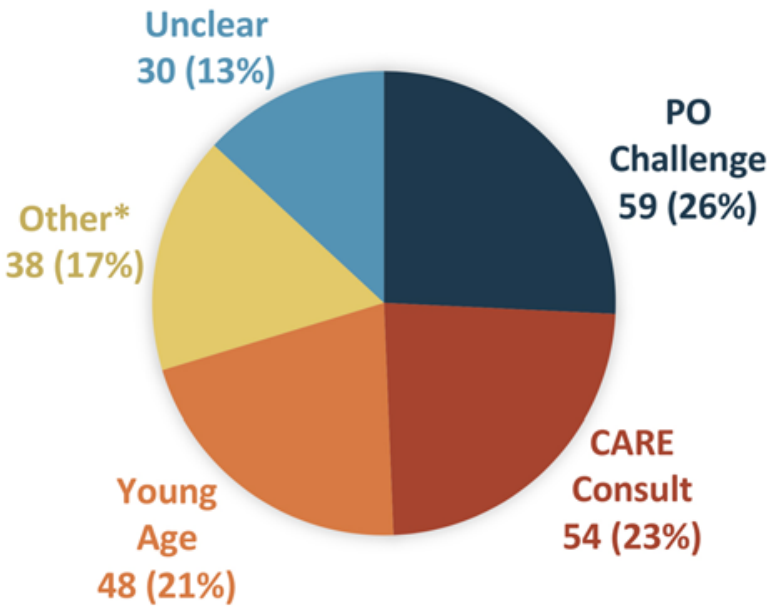

\footnotetext{
*Monitoring (PCPS) - $13(6 \%)$

Monitoring (other) -7 (3\%)

Neurological symptoms -7 (3\%)

NAT workup - $3(1 \%)$

High velocity injury $-2(<1 \%)$

ET tube $-1(<1 \%)$

Further evaluation $-3(1 \%)$

Pain $-1(<1 \%)$

Fracture displacement (minimal) $-1(<1 \%)$
}

FIG. 1. Reasons patients were admitted to the hospital from the ED. ET $=$ endotracheal; $\mathrm{PCPS}=$ pneumocephalus. Figure is available in color online only.

mission rate. However, presenting to the hospital more than 24 hours after injury was predictive of a decreased admission rate.

There were 21 patients $(4.1 \%)$ who revisited the ED following their initial discharge. Over half of these revisits were attributable to vomiting (52\%). Of those patients who did revisit the ED, $16(76.2 \%)$ had been initially discharged directly from the ED, whereas the other $5(23.8 \%)$ had been previously admitted to the hospital. Eleven $(52.4 \%)$ of the 21 patients had been treated with ondansetron prior to their initial discharge. The only significant predictor of revisits following multivariable analysis was ondansetron

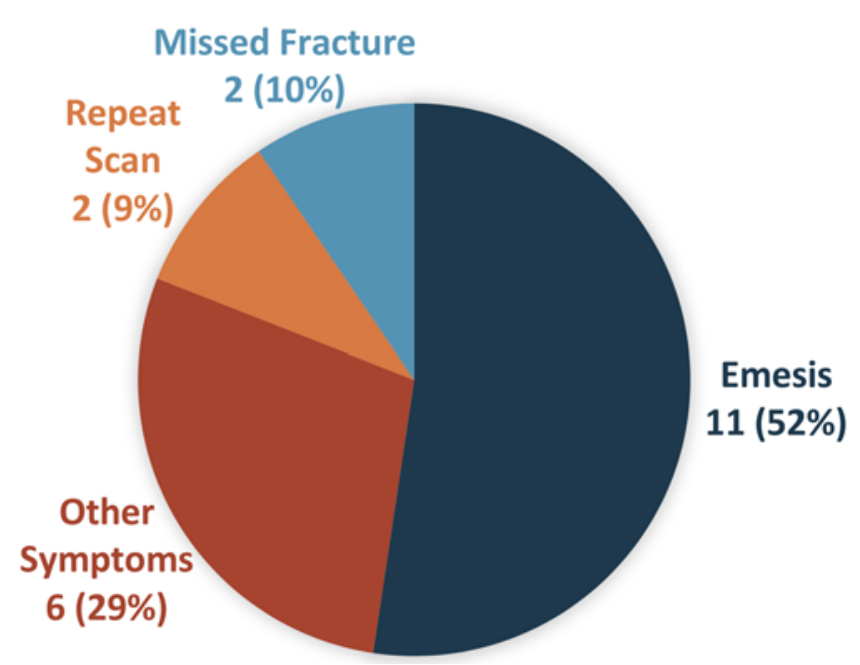

FIG. 2. Breakdown of reasons patients revisited the ED within 72 hours of leaving the hospital following initial skull fracture workup and management. Figure is available in color online only.
TABLE 2. The $p$ values for univariable analysis of the primary study outcomes

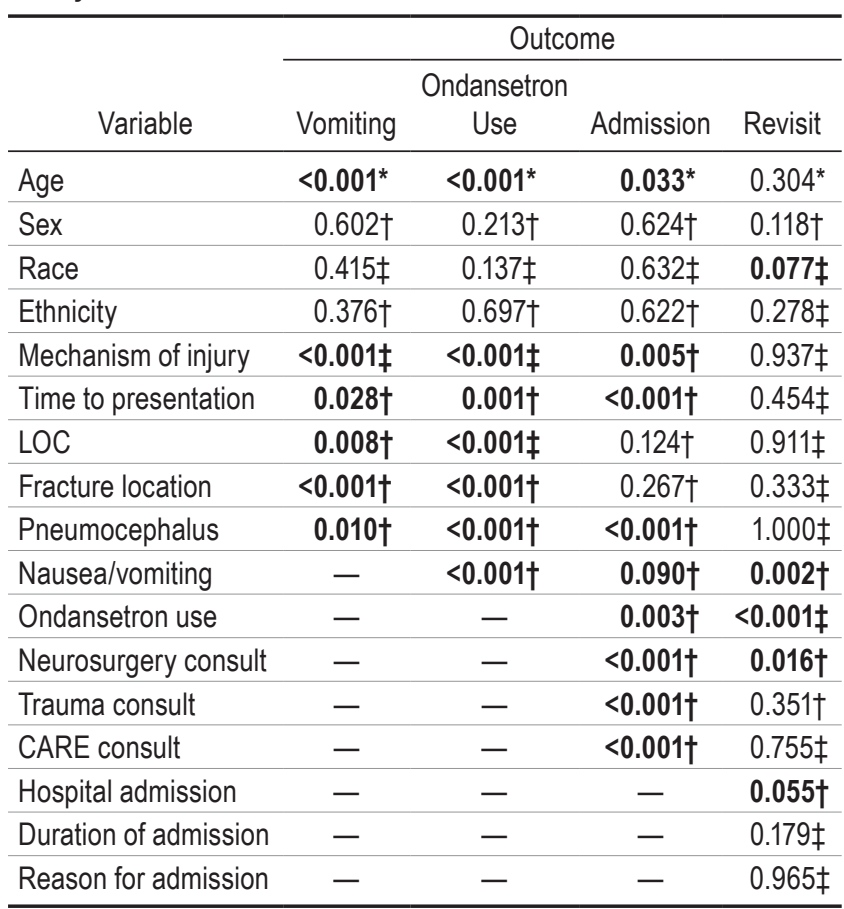

LOC $=$ loss of consciousness

Boldface type indicates statistical significance and variables included in the multivariable model for the associated outcome.

* Univariable logistic regression.

† Pearson's chi-square test.

‡ Fisher's exact test (used when there is an expected cell count $<5$ ).

use at the initial admission (OR 5.05, $\mathrm{p}=0.009$ ). While vomiting alone was the reason for a majority of revisits, it was not an independent predictor of revisits in the multivariable analysis $(\mathrm{OR} 1.57, \mathrm{p}=0.457)$.

\section{Discussion}

Overall, the cohort described in this study is similar to those from prior studies of pediatric ISFs. At least $76 \%$ of patients were injured from a fall (with $62 \%$ having a lowerimpact fall from $\leq 3$ feet), which is in line with prior injury profiles. ${ }^{2,5,9,12,14,16}$ Similarly, parietal bone fractures were the most common, which has been shown to be closely related to falling. ${ }^{2}$ Given our study and the literature, our cohort seems to be a reasonably generalizable population of pediatric patients with ISFs.

The overall admission rate for patients in this study was $44 \%$, which is lower than rates in most prior studies that range from $57 \%$ to $83 \%$ (with a few outliers). $4,6,9,11,12$, $14,16,20$ Of those cases admitted, $26 \%$ were PO challenges and $23 \%$ were for CARE consults. Another $17 \%$ were admitted for more specific reasons, such as neurological symptoms or pneumocephalus monitoring. That said, a number of patients in this cohort may have been admitted unnecessarily. The remaining third of the cohort was admitted for either young age $(21 \%)$ or unclear reasons $(13 \%)$. As in prior studies, all of these patients recovered well from their injury with no further decline or complica- 
TABLE 3. Significant predictors of each primary outcome in the multivariable logistic regression

\begin{tabular}{lcr}
\hline \multicolumn{1}{c}{ Outcome } & OR & p Value \\
\hline Vomiting & & \\
\hline Age in mos & $1.01 / \mathrm{mo}$ & $\mathbf{0 . 0 0 2}$ \\
\hline Occipital location of fracture & 4.05 & $<0.001$ \\
\hline Ondansetron use & & \\
\hline Age in mos & $1.02 / \mathrm{mo}$ & $<0.001$ \\
\hline MVC as mechanism of injury & 4.49 & $\mathbf{0 . 0 2 6}$ \\
\hline Principal location of fracture & & \\
\hline Occipital & 2.66 & 0.017 \\
\hline Temporal & 4.44 & $\mathbf{0 . 0 1 3}$ \\
\hline Nausea/vomiting & 14.42 & $<0.001$ \\
\hline Hospital admission & & \\
\hline Time from injury to presentation $>24$ hrs & 0.15 & $<0.001$ \\
\hline Neurosurgery consult & 4.45 & $<0.001$ \\
\hline Trauma consult & 32.51 & $<0.001$ \\
\hline CARE consult & 31.13 & $<0.001$ \\
\hline ED revisit & & \\
\hline Ondansetron use & 5.05 & $\mathbf{0 . 0 0 9}$ \\
\hline
\end{tabular}

Boldface type indicates statistical significance.

tions. Thus, it seems likely that admission solely for young age or general observation is not required.

While vomiting was the most common indication for admission in our cohort, the role of vomiting and antiemetic use in skull fractures is unclear in the literature. Nee et al. showed that posttraumatic vomiting was associated with a fourfold increase in skull fracture rates; however, others have shown that vomiting rates are not associated with any one aspect of head injury. ${ }^{7,13}$ In our study, occipital fractures were significantly more likely to result in vomiting, which confirmed one of our hypotheses. The exact pathophysiological reason for this finding is unclear. It could simply be related to the force of the trauma needed to cause occipital fractures. That said, none of the injury profiles (e.g., falls from $>3$ feet) was independently associated with vomiting. This finding could also be related to the location of injury relative to the cerebellum, brainstem, or vestibular apparatus in the posterior fossa. In turn, PO challenge was one of the primary indications for both hospital admission and revisit following ED discharge. The apparent role that fracture location plays in the development of vomiting could be something to consider when making admission decisions.

While vomiting was the most common indication for both admission and revisit, it was not independently predictive of either, which negates our remaining study hypothesis. On the other hand, ondansetron use was highly associated with revisits following discharge but not with admission. Mixed findings have been reported on this relationship, with two studies showing opposite effects of ondansetron use on the 72-hour revisit rate. ${ }^{8,17}$ In this population, ondansetron use is perhaps masking vomiting in the hospital, leading to ED revisits once the vomiting recurs. In any case, it is apparent that there is a nuanced relationship between ondansetron use and ED revisits. If a patient has nausea/vomiting that is severe enough to require ondansetron use, they likely require some form of observation (whether in the form of hospital admission or ED observation). That said, ondansetron use should be limited to those patients who require it and not applied as a universal "nausea prophylaxis" or as a method to expedite discharge, as this is likely to raise revisit rates. Further, patient education about the effects of ondansetron and postconcussive vomiting could help reduce unnecessary revisits that result if mild vomiting recurs. Some details of the relationship between ondansetron use and ED revisits, such as the timing of ondansetron administration, could not be assessed in this study. That said, they are the subject of future work.

Interestingly, the only positive predictors of admission rates were consults to each of the three services investigated (neurosurgery, trauma surgery, and CARE). The reason for trauma surgery consult is likely that trauma surgery is often the admitting service at our institution, so the recommendation to admit is often proffered before trauma surgery is consulted. Certainly there is some variability in the ED evaluation of these patients - in some cases, trauma surgery is consulted in response to a patient presenting via ambulance following a traumatic event with a significant mechanism of injury (e.g., a motor vehicle accident creating a level I or level II trauma activation), rather than solely serving as the admitting service. CARE consults reflect patients with suspected NAT, which we believe is both an appropriate and expected reason for admission as well as neurosurgical consultation.

On the contrary, the relationship between neurosurgery consults and higher admission rates was unexpected. Perhaps neurosurgery consults are serving as a proxy for perceived injury severity as none of the patients in our cohort had depressed fractures or other significant injuries. It is also common for patients who sustain higher mechanisms of injury and present as a level I or II trauma activation to be admitted per the institution-dependent trauma surgery service guidelines and subsequently receive neurosurgical consultation to help guide management while in-house. This could inspire a reanalysis of existing trauma surgery protocols, but this is outside the scope of this article. In any case, the complex relationship among services involved in the care of these patients could indicate that patients would benefit from an objective admission protocol.

It is also important to consider each patient's social situation, which frequently plays a significant role in the decision to admit or discharge. Unfortunately, outside of NAT suspicion, we were unable to assess each patient's specific social characteristics and whether or not they played a role in admission. However, given that $13 \%$ of the patients were admitted for unclear reasons, it is both possible and likely that some of these admissions were attributable to social factors, such as a lack of transportation home or a late hour of presentation (in addition to cases of NAT suspicion, which was a separate category). Alongside clinical factors, social issues need to be considered when determining whether a patient is appropriate for discharge.

Overall, a well-established protocol to avoid unnecessary admissions and revisits would be beneficial in reduc- 
ing both admission rates and overall hospital costs. This study is in agreement with prior reports that unrelenting vomiting/PO challenge, NAT suspicion, and other neurological symptoms are important factors that often warrant admission.,3,15,16,19 In addition to these factors, occipital fracture location and recent ondansetron use should be considered, as they are likely related to recurrent vomiting. On the contrary, admission for young age and inpatient observation (in the absence of other injuries or concerns) is generally not warranted, as patients in these cases are highly unlikely to clinically decline or develop further complications. Patients who are deemed suitable for discharge from a clinical standpoint should also be cleared from a social perspective at the discretion of the ED. While a protocol for our institution has not yet been fully validated, a preliminary protocol has been developed in collaboration with other services and a prospective trial is underway. Results of this study and the specifics of the corresponding trial will be detailed when completed.

The present study is not without limitations. Because it is a retrospective study, there are certain variables that could not be accounted for. While disease severity was somewhat controlled for by using mechanism of injury and exclusion criteria selecting only for ISFs, this does not cover the range of clinical aspects to consider when deciding to admit or discharge. Additionally, this study took place at a level I trauma center that frequently receives patients from surrounding hospitals and clinics. The medical records received from these providers are often incomplete and, as a result, may leave out details such as vomiting and ondansetron use, leading to underestimation of these variables. Given that this study includes both infants and older children/adolescents, it is possible that there are unmeasured confounding factors between the two age groups. We attempted to control for these by including age and other possible differences, such as mechanism of injury, in the multivariable model. Furthermore, vomiting can be misinterpreted as normal spit-up by an inexperienced pediatric provider, particularly among infant patients. Which type of provider classifies vomiting in the field-for example, a rural emergency medical technician versus a pediatrics-trained paramedic_could be considered in future studies. Finally, we were unable to assess which patients were admitted as inpatients versus those who were held for 23-hour observation, which likely has further cost implications. Thus, a prospective study based on the results of this study is under development at our institution to further refine and evaluate an objective admission protocol and assess the effects of such a protocol on the cost of patient care.

\section{Conclusions}

Pediatric ISFs are relatively common injuries that have a very low risk of further clinical decline. Despite this, a number of patients with these injuries are unnecessarily admitted for reasons such as young age or general observation. In this study, we identified occipital fracture location as a predictor of vomiting that could be considered, along with neurological symptoms, vomiting, and NAT suspicion, in the admission decision-making. Moreover, recent ondansetron use was a significant predictor of revisits following ED discharge, whereas vomiting alone was not. This finding may warrant further clinical investigation to reduce revisit rates. An objective and well-established protocol would be critical in streamlining decision-making, reducing unnecessary admissions, lowering ED revisit rates, and decreasing the excess cost associated with unnecessary hospitalizations.

\section{Acknowledgments}

Study data were collected and managed using REDCap electronic data capture tools hosted at Vanderbilt University. REDCap (Research Electronic Data Capture) is a secure, Web-based application designed to support data capture for research studies, providing 1) an intuitive interface for validated data entry, 2) audit trails for tracking data manipulation and export procedures, 3 ) automated export procedures for seamless data downloads to common statistical packages, and 4) procedures for importing data from external sources.

\section{References}

1. Addioui A, Saint-Vil D, Crevier L, Beaudin M: Management of skull fractures in children less than 1 year of age. J Pediatr Surg 51:1146-1150, 2016

2. Adepoju A, Adamo MA: Posttraumatic complications in pediatric skull fracture: dural sinus thrombosis, arterial dissection, and cerebrospinal fluid leakage. J Neurosurg Pediatr 20:598-603, 2017

3. Arrey EN, Kerr ML, Fletcher S, Cox CS Jr, Sandberg DI: Linear nondisplaced skull fractures in children: who should be observed or admitted? J Neurosurg Pediatr 16:703-708, 2015

4. Blackwood BP, Bean JF, Sadecki-Lund C, Helenowski IB, Kabre R, Hunter CJ: Observation for isolated traumatic skull fractures in the pediatric population: unnecessary and costly. J Pediatr Surg 51:654-658, 2016

5. Bonfield CM, Naran S, Adetayo OA, Pollack IF, Losee JE: Pediatric skull fractures: the need for surgical intervention, characteristics, complications, and outcomes. J Neurosurg Pediatr 14:205-211, 2014

6. Bressan S, Marchetto L, Lyons TW, Monuteaux MC, Freedman SB, Da Dalt L, et al: A systematic review and metaanalysis of the management and outcomes of isolated skull fractures in children. Ann Emerg Med 71:714-724.e2, 2018

7. Brown FD, Brown J, Beattie TF: Why do children vomit after minor head injury? J Accid Emerg Med 17:268-271, 2000

8. Green-Hopkins I, Monuteaux MC, Lee L, Nigrovic L, Mannix R, Schutzman S: Use of ondansetron for vomiting after head trauma. Pediatr Emerg Care [epub ahead of print], 2017

9. Greenes DS, Schutzman SA: Infants with isolated skull fracture: what are their clinical characteristics, and do they require hospitalization? Ann Emerg Med 30:253-259, 1997

10. Harris PA, Taylor R, Thielke R, Payne J, Gonzalez N, Conde JG: Research electronic data capture (REDCap) - a metadata-driven methodology and workflow process for providing translational research informatics support. J Biomed Inform 42:377-381, 2009

11. Mannix R, Monuteaux MC, Schutzman SA, Meehan WP III, Nigrovic LE, Neuman MI: Isolated skull fractures: trends in management in US pediatric emergency departments. Ann Emerg Med 62:327-331, 2013

12. Metzger RR, Smith J, Wells M, Eldridge L, Holsti M, Scaife ER, et al: Impact of newly adopted guidelines for management of children with isolated skull fracture. J Pediatr Surg 49:1856-1860, 2014 
13. Nee PA, Hadfield JM, Yates DW, Faragher EB: Significance of vomiting after head injury. J Neurol Neurosurg Psychiatry 66:470-473, 1999

14. Powell EC, Atabaki SM, Wootton-Gorges S, Wisner D, Mahajan P, Glass T, et al: Isolated linear skull fractures in children with blunt head trauma. Pediatrics 135:e851-e857, 2015

15. Reuveni-Salzman A, Rosenthal G, Poznanski O, Shoshan Y, Benifla M: Evaluation of the necessity of hospitalization in children with an isolated linear skull fracture (ISF). Childs Nerv Syst 32:1669-1674, 2016

16. Rollins MD, Barnhart DC, Greenberg RA, Scaife ER, Holsti M, Meyers RL, et al: Neurologically intact children with an isolated skull fracture may be safely discharged after brief observation. J Pediatr Surg 46:1342-1346, 2011

17. Sturm JJ, Simon HK, Khan NS, Hirsh DA: The use of ondansetron for nausea and vomiting after head injury and its effect on return rates from the pediatric ED. Am J Emerg Med 31:166-172, 2013

18. Tunik MG, Powell EC, Mahajan P, Schunk JE, Jacobs E, Miskin M, et al: Clinical presentations and outcomes of children with basilar skull fractures after blunt head trauma. Ann Emerg Med 68:431-440.e1, 2016

19. Vogelbaum MA, Kaufman BA, Park TS, Winthrop AL: Management of uncomplicated skull fractures in children: is hospital admission necessary? Pediatr Neurosurg 29:96-101, 1998

20. Williams DC, Russell WS, Andrews AL, Simpson KN, Basco WT Jr, Teufel RJ II: Management of pediatric isolated skull fractures: a decision tree and cost analysis on emergency department disposition strategies. Pediatr Emerg Care 34:403-408, 2018

\section{Disclosures}

This research was supported in part by the Surgical Outcomes Center for Kids at Monroe Carell Jr. Children's Hospital at Vanderbilt and through the Section for Surgical Sciences at Vanderbilt University Medical Center.

\section{Author Contributions}

Conception and design: Dallas, Reynolds, Wellons, Shannon, Bonfield. Acquisition of data: Dallas, Mercer, Reynolds. Analysis and interpretation of data: Dallas, Reynolds, Wellons, Shannon, Bonfield. Drafting the article: Dallas, Mercer, Reynolds. Critically revising the article: Dallas, Reynolds, Wellons, Shannon, Bonfield. Reviewed submitted version of manuscript: all authors. Approved the final version of the manuscript on behalf of all authors: Dallas. Statistical analysis: Dallas. Administrative/ technical/material support: Shannon. Study supervision: Wellons, Shannon, Bonfield.

\section{Correspondence}

Jonathan Dallas: Vanderbilt University School of Medicine, Nashville,TN.jonathan.c.dallas@vanderbilt.edu. 